\title{
GEVREY CLASS SEMIGROUPS ARISING FROM ELASTIC SYSTEMS WITH GENTLE DISSIPATION: THE CASE $0<\alpha<\frac{1}{2}$
}

\author{
SHUPING CHEN AND ROBERTO TRIGGIANI
}

(Communicated by Barbara L. Keyfitz)

\begin{abstract}
Let $A$ (the elastic operator) be a positive, self-adjoint operator with domain $\mathscr{D}(A)$ in the Hilbert space $X$, and let $B$ (the dissipation operator) be another positive, self-adjoint operator satisfying $\rho_{1} A^{\alpha} \leq B \leq \rho_{2} A^{\alpha}$ for some constants $0<\rho_{1}<\rho_{2}<\infty$ and $0<\alpha \leq 1$. Consider the operator

$$
\mathscr{A}_{B}=\left|\begin{array}{cc}
0 & I \\
-A & -B
\end{array}\right|
$$

(corresponding to the elastic model $\ddot{x}+B \dot{x}+A x=0$ written as a first order system), which (once closed) is plainly the generator of a strongly continuous semigroup of contractions on the space $E=\mathscr{D}\left(A^{1 / 2}\right) \times X$. In [C-T.1] [C-T.3] we showed that, for $\frac{1}{2} \leq \alpha \leq 1$, such a semigroup is analytic (holomorphic) on $E$ on a triangular sector of $\mathbf{C}$ containing the positive real axis and, moreover, that the property of analyticity is false for $0<\alpha<\frac{1}{2}$, say for $B=A^{\alpha}$. We now complete the description of $\mathscr{A}_{B}$ in the range $0<\alpha<\frac{1}{2}$ by showing that such semigroup is in fact of Gevrey class $\delta>1 / 2 \alpha$, hence differentiable on $E$ for all $t>0$.
\end{abstract}

\section{INTRODUCTION AND STATEMENT OF MAIN RESULT}

1.1. Introduction. Throughout this note we shall assume that:

(H.1) $A$ (the elastic operator) is a self-adjoint operator on a Hilbert space $X$, strictly positive, with dense domain $\mathscr{D}(A)$.

(H.2) $B$ (the dissipation operator) is, for the time being, a positive, selfadjoint operator on $X$, likewise with dense domain $\mathscr{D}(B)$ in $X$.

Received by the editors June 26, 1989 and, in revised form, November 2, 1989; this paper was presented by the second author at the 27th IEEE Conference on Decision and Control, 'held in Austin, Texas, December 7-9, 1988.

1980 Mathematics Subject Classification (1985 Revision). Primary 47A50, 47A55; Secondary 35B40.

Research of the first author was supported by the National Natural Science Foundation of China.

Part of this research was carried out while the second named author was a visiting scientist at the Interdisciplinary Center for Applied Mathematics, Virginia Polytechnic Institute and State University, which is partially funded under DARPA Contract No. F49620-87-C-0116. Partial support by the Air Force Office of Scientific Research under grant AFOSR-87-0321 is gratefully acknowledged. 
The object of our interest is the abstract differential equation

$$
\ddot{x}+B \dot{x}+A x=0 \text { on } X,
$$

or

$$
\frac{d}{d t}\left|\begin{array}{c}
x \\
\dot{x}
\end{array}\right|=\mathscr{A}_{B}\left|\begin{array}{c}
x \\
\dot{x}
\end{array}\right| \quad \text { on } \quad E=\mathscr{D}\left(A^{1 / 2}\right) \times X
$$

$$
\mathscr{A}_{B}=\left|\begin{array}{cc}
0 & I \\
-A & -B
\end{array}\right| \text { with domain } \mathscr{D}\left(\mathscr{A}_{B}\right) \text { containing } \mathscr{D}(A) \times \mathscr{D}(B),
$$

where the inner product on $E$ is defined by

$$
\left(\left[x_{1}, x_{2}\right],\left[y_{1}, y_{2}\right]\right)_{E}=\left(A^{1 / 2} x_{1}, A^{1 / 2} y_{1}\right)_{X}+\left(x_{2}, y_{2}\right)_{X} .
$$

The operator $\mathscr{A}_{B}$ is densely defined and dissipative on $E$, hence closeable on $E$, and we shall use the same symbol $\mathscr{A}_{B}$ to denote its closure. The LumerPhillips theorem then readily shows that $\mathscr{A}_{B}$ generates a strongly continuous semigroup of contractions on $E$, denoted by $e^{\mathscr{A}_{B} t}$, e.g., [C-T.1, C-T.2]. Stimulated by two conjectures raised in [C-R.1], we have already studied in [C-T.1, C-T.3] problem (1.1), or (1.2), under the additional hypothesis that $B$ is "comparable to $A^{\alpha}$,, with emphasis on the range $\frac{1}{2} \leq \alpha \leq 1$. For the purposes of the present note, we shall recall the following version of the results described in [C-T.1, C-T.3]. Our hypothesis that $B$ is comparable to $A^{\alpha}$ is as follows.

(H.3) There exist two constants $0<\rho_{1}, \rho_{2}<\infty$ and a constant $0<\alpha \leq 1$ such that

$$
\rho_{1} A^{\alpha} \leq B \leq \rho_{2} A^{\alpha}
$$

i.e.,

$$
\rho_{1}\left(A^{\alpha} x, x\right)_{X} \leq(B x, x)_{X} \leq \rho_{2}\left(A^{\alpha} x, x\right)_{X}, \quad x \in \mathscr{D}\left(B^{1 / 2}\right)=\mathscr{D}\left(A^{\alpha / 2}\right)
$$

Condition $(1.5 \mathrm{~b})$ is actually equivalent to $\mathscr{D}\left(B^{1 / 2}\right)=\mathscr{D}\left(A^{\alpha / 2}\right)$, since then $B^{1 / 2} A^{-\alpha / 2}$ and $A^{\alpha / 2} B^{-1 / 2}$ are bounded operators on $X$.

Theorem A. [C-T.1, C-T.2]. Assume the standing hypotheses (H.1), (H.2) and, in addition, hypothesis (H.3) with $\frac{1}{2} \leq \alpha \leq 1$ fixed in (1.5). Then, the strongly continuous semigroup of contractions $\exp \left(\mathscr{A}_{B} t\right)$ generated by the operator $\mathscr{A}_{B}$ in (1.3) (once closed) is also analytic (holomorphic) on $E=\mathscr{D}\left(A^{1 / 2}\right) \times X$. In particular, if $\alpha=\frac{1}{2}$, then $\exp \left(\mathscr{A}_{B} t\right)$ is a strongly continuous, analytic semigroup of contractions also on each space

$$
E_{\theta}=\mathscr{D}\left(A^{3 / 4-\theta / 2}\right) \times \mathscr{D}\left(A^{1 / 4-\theta / 2}\right), \quad 0 \leq \theta \leq 1 .
$$

In (1.6) we have used the convention that $\mathscr{D}\left(A^{-\beta}\right)=\left[\mathscr{D}\left(A^{\beta}\right)\right]^{\prime}, \beta>0$, the dual space of $\mathscr{D}\left(A^{\beta}\right)$ with respect to the $X$-topology, where

$$
\|x\|_{\mathscr{L}\left(A^{\beta}\right)}=\left\|A^{\beta} x\right\|_{X} ; \quad\|x\|_{\left[\mathscr{U}\left(A^{\beta}\right)\right]^{\prime}}=\left\|A^{-\beta} x\right\|_{X} .
$$


A fortiori, the spectrum determined growth assumption is satisfied for $\mathscr{A}_{B}$ [T.1] and there are constants $\delta=-\sup \operatorname{Re} \sigma\left(\mathscr{A}_{B}\right)>0, \sigma\left(\mathscr{A}_{B}\right)$ being the spectrum of $\mathscr{A}_{B}$, and $M$ such that

$$
\left\|e^{\mathscr{A}_{B} t}\right\|_{\mathscr{L}(E)} \leq M e^{-\delta t}, \quad t \geq 0,
$$

and similarly on $\mathscr{L}\left(E_{\theta}\right)$ for $\alpha=\frac{1}{2}$.

Remark 1.1. In Theorem $\mathrm{A}$, the assumption that $B$ is self-adjoint is unnecessary. Indeed, the conclusions of Theorem $A$ for $1 / 2 \leq \alpha \leq 1$ hold true if $B$ is closed and satisfies the conditions $\left(\right.$ H. $\left.3^{\prime}\right)$ :

$$
\begin{gathered}
\rho_{1}\left(A^{\alpha} x, x\right) \leq \operatorname{Re}(B x, x) \leq \rho_{2}\left(A^{\alpha} x, x\right), \quad x \in \mathscr{D}\left(A^{\alpha / 2}\right) \\
|\operatorname{Im}(B x, x)| \leq K \operatorname{Re}(B x, x), \quad x \in \mathscr{D}\left(A^{\alpha / 2}\right)
\end{gathered}
$$

which generalize $(1.5 b)$. This extension requires only a minor variation of the proof of, say, [C-T.2; Lemma 4.2], which will be indicated in the proof of Lemma 3.1 of the present paper. Another proof is given in [C-T.3]. Here, we note that condition $(1.9 \mathrm{~b})$ is necessary for analyticity of $\exp \left(\mathscr{A}_{B} t\right)$. In fact, in [C-T.3] it is shown that if $B=A^{\alpha}+i A^{\beta}$ with $\alpha<\beta$, where, say, $A$ has compact resolvent, then the eigenvalues $\lambda_{n}$ of $\mathscr{A}_{B}$ satisfy $\left|\operatorname{Im} \lambda_{n} / \operatorname{Re} \lambda_{n}\right| \rightarrow \infty$ and thus $\exp \left(\mathscr{A}_{B} t\right)$ cannot be analytic or even differentiable on $E$.

Remark 1.2. The following (less interesting) results hold true as well, say when $B$ is positive self-adjoint:

(i) $\exp \left(\mathscr{A}_{B} t\right)$ is analytic on $E$, if $\rho_{1} A \leq B$; equivalently, if $\mathscr{D}\left(B^{1 / 2}\right) \subset$ $\mathscr{D}\left(A^{1 / 2}\right)$; more generally, if $B$ not necessarily self-adjoint, satisfies $\rho_{1}(A x, x) \leq \operatorname{Re}(B x, x), x \in \mathscr{D}(B)$, as well as $(1.9 \mathrm{~b})$;

(ii) $\exp \left(\mathscr{A}_{B} t\right)$ is analytic on $E$ if $\rho_{1} A^{1 / 2} \leq B$, equivalently if $\mathscr{D}\left(B^{1 / 2}\right) \subset$ $\mathscr{D}\left(A^{1 / 4}\right)$, and $A$ and $B$ commute.

These results can be proved, say, by the methods in [C-T.2, §4].

The desirable exponential (uniform) decay (1.8) is here merely obtained afortiori as a by-pass product of analyticity. A control theoretic reformulation of the above decay result (1.8) is that the control function $u=-B x$ in feedback form uniformly stabilizes the original conservative system $\ddot{x}+A x=u$, see Remark 1.3. There is at present a revival of investigations on finding general abstract conditions which ensure exponential (uniform) decay of s. c. semigroups [L.1].

References [C-T.1, C-T.3] present, collectively, several proofs of the above results. (The case $\frac{1}{2}<\alpha \leq 1$ offers new genuine difficulties over the case $\alpha=\frac{1}{2}$.) The above results established a fortiori two conjectures raised in [CR.1] which referred to the case $\alpha=\frac{1}{2}$. In [C-T.1, C-T.2] the following results are likewise noted: (i) in the range $0<\alpha<\frac{1}{2}$, the conclusion of Theorem A is generally false in that the semigroup $\exp \left(\mathscr{A}_{B} t\right)$ is generally not analytic (classes of counterexamples provided); (ii) in the range $0<\alpha<\frac{1}{2}$ and for the 
special case $B=2 \rho A^{\alpha}, 0<\rho<\infty$, the corresponding semigroup is in fact differentiable for all $t>0$ on $E$ (but not analytic here): $\exp \left(\mathscr{A}_{B} t\right) E \subset \mathscr{D}\left(\mathscr{A}_{B}\right)$, $t>0$, see [P.1] and Remark A.2 in [C-T.2]. This result will be reviewed in $\S 2$.

1.2. Statement of the main result. The aim of the present note is to focus attention on the range $0<\alpha<\frac{1}{2}$ in assumption (H.3) and complete the description of the semigroup $\exp \left(\mathscr{A}_{B} t\right)$ as a function of $\alpha$ by showing the following result.

Theorem 1.1. Assume the standing assumptions (H.1), (H.2) and assumption (H.3) with $0<\alpha<\frac{1}{2}$ in (1.5). Then the resolvent $R\left(\cdot, \mathscr{A}_{B}\right)$ of the operator $\mathscr{A}_{B}$ in (1.3), which (once closed) generates a strongly continuous semigroup of contractions on $E$, satisfies

$$
\lim _{|\tau| \rightarrow \infty}|\tau|^{2 \alpha}\left\|R\left(i \tau, \mathscr{A}_{B}\right)\right\|_{\mathscr{L}(E)}=c<\infty, \quad \tau \in R
$$

Thus, [T.4, Theorem 4], [P.1, Theorem 4.9, p. 57] the semigroup $\exp \left(\mathscr{A}_{B} t\right)$, is moreover, of Gevrey class $\delta>1 / 2 \alpha$, hence differentiable for all $t>0$ on $E$. $A$ fortiori, the spectrum determined growth assumption is satisfied for $\mathscr{A}_{B}$ [T.1] and there are constants $\delta>0$ and $M$ as in Theorem $A$ such that inequality (1.8) holds true.

Thus, the transition from the group case $\alpha=0$ (unitary, if $B=0$ ) to the analytic semigroup case $\frac{1}{2} \leq \alpha \leq 1$ passes through the intermediate stage of Gevrey semigroups for $0<\alpha<\frac{1}{2}$. Gevrey regularity involves bounds on the $n$th derivatives which are similar to, but weaker than, the bounds on the $n$th derivatives in the analytic case.

An s.c. semi-group $T(t)$ is of Gevrey class $\delta$ for $t>t_{0}$ if $T(t)$ is infinitely differentiable for $t \in\left(t_{0}, \infty\right)$ and for every compact $\mathscr{K} \subset\left(t_{0}, \infty\right)$ and each $\theta>0$, there exists a constant $C=C(\theta, \mathscr{K})$ such that $\left\|T^{(n)}(t)\right\| \leq C \theta^{n}(n !)^{\delta}$ for all $t \in \mathscr{K}$ and $n=0,1,2, \ldots$ see [T.4], where a theory is developed which parallels the theory of differentiable semi-groups in [P.1].

The proof given below, though inspired by our previous work on analyticity [C-T.2, §4], encounters new features peculiar to the Gevrey case. In particular, we shall crucially use the property that the generator of the Gevrey semigroup which arises in the special case $B=2 \rho A^{\alpha}, 0<\alpha<\frac{1}{2}$, has its spectrum $\left\{\lambda_{n}^{+,-}\right\}$which displays only a polynomial growth (for fixed $\alpha$ ) of the function $\left|\operatorname{Re} \lambda_{n}^{+,-}\right| \rightarrow\left|\operatorname{Im} \lambda_{n}^{+,-}\right|$, a much better behavior than the exponential growth allowed for the general class of differentiable semigroups. This will result in the stronger property of the corresponding resolvent operator expressed by Corollary 2.3 when $B=2 \rho A^{\alpha}$. This in turn will allow us to prove a similar property ((1.10) above) for the resolvent operator of $\mathscr{A}_{B}$ for a general $B$ which satisfies (1.5), or, more generally, (1.9). The latter property (1.10) is precisely a sufficient condition for $\exp \left(\mathscr{A}_{B} t\right)$ to be Gevrey on $E$ for all $t>0$. (Our strategy [CT. $2, \S 4]$ in the analytic case was somewhat different in that the necessary and 
sufficient condition of analyticity for the special case $B=2 \rho A^{\alpha}, \frac{1}{2} \leq \alpha \leq$ 1 , was transferred into the necessary and sufficient condition for a general $B$ satisfying (1.5), or more generally, (1.9).)

Remark 1.3 . One can readily show that, with the notation

$$
\mathscr{P}=\left|\begin{array}{cc}
0 & 0 \\
0 & -B
\end{array}\right|, \quad \mathscr{A}_{0}=\left|\begin{array}{cc}
0 & I \\
-A & 0
\end{array}\right|,
$$

the perturbation $\mathscr{P}$ is $\mathscr{A}_{0}$-bounded on $E$ for $0<\alpha \leq \frac{1}{2}$; indeed, is $\mathscr{A}_{0}$ compact on $E\left[\mathrm{~K} .2\right.$, p. 194] for $0<\alpha<\frac{1}{2}$. Thus, in view of Theorem 1.1, the second order problem $\ddot{x}+A x=u$, which is conservative on $E$ for $u \equiv 0$, is uniformly stabilized on $E$ by the feedback operator $u=-B \dot{x}, B$ as in (1.5) for $0<\alpha<\frac{1}{2}$, which yields a relatively compact perturbation $\mathscr{P}$ on $E$.

By contrast, a conservative problem cannot be uniformly stabilized by a relatively bounded perturbation of finite rank [T.3, Theorem 2.1]. Thus, the result just quoted from [T.3] on the lack of uniform stabilization is sharp also in the sense that the finite rank assumption on the class of relatively bounded perturbations cannot be replaced with the more relaxed class of just relatively compact perturbations. (In addition, as observed in [T.3], the assumption that the class of perturbations be only relatively bounded and not any worse, is also an optimal result for the lack of uniform stabilization of a conservative problem modeled by an operator like $\mathscr{A}$.)

Remark 1.4. In its original version, our paper claimed less than it showed. Indeed, it only claimed that the s.c. contraction semigroup $\exp \left(\mathscr{A}_{B} t\right)$ is differentiable on $E$ for all $t>0$. However, the arguments in the paper actually showed the uniform estimate (1.10). This was stated explicitly in the case $B=2 \rho A^{\alpha}$, or $\mathscr{A}_{B}=\mathscr{A}_{\rho \alpha}$, as in Corollary 2.3 below. In its original version the subsequent arguments of $\S 3$ dropped the polynomial dependence $|\tau|^{2 \alpha}$ obtained for $R\left(i \tau, \mathscr{A}_{\rho \alpha}\right)$ in favor of the weaker logarithmic dependence $\log |\tau|$ for $R\left(i \tau, \mathscr{A}_{B}\right)$, for this was all that was needed to fall into the known sufficient condition for differentiability as in Pazy [P.1, Corollary 4.10, p. 58]. If the polynomial dependence is retained, the very same argument as the original one yields instead the uniform estimate $(1.10)$ in the general case $\mathscr{A}_{B}$.

In October 1989, Steve Taylor (Georgia Institute of Technology) kindly pointed out to us his recent Ph.D. work [T.4], where a theory of semigroups of Gevrey class $\delta$ is constructed, which is the counterpart of the theory of differentiable semigroups as in Pazy [P.1]. In particular, S. Taylor shows [T.4, Theorem 4] that the uniform estimate (1.10) is, in fact, a sufficient condition for the semigroup to be of Gevrey class $\delta>\frac{1}{2 \alpha}$. (This result is the counterpart with $|\tau|^{2 \alpha}$ instead of $\log |\tau|$ of the sufficient condition [P.1, Theorem 4.9, p. 57] for differentiable semigroups, to which we had originally appealed.) S. Taylor also noted that our original proof yields the uniform estimate $(1.10)$ for general $\mathscr{A}_{B}$, thus providing a nontrivial class of Gevrey semigroups. Thus, by invoking S. Taylor [T.4] rather than Pazy [P.1], we can uplift our original claim and 
conclude that the semigroup in Theorem 1.1 is actually of Gevrey class $\delta>\frac{1}{2 \alpha}$. We wish to thank S. Taylor for bringing his work [T.4] to our attention.

$$
\text { 2. The CASE } B=2 \rho A^{\alpha}, 0<\alpha<\frac{1}{2} \text {. }
$$

Let

$$
B=2 \rho A^{\alpha}, \quad 0<\rho<\infty, 0<\alpha<\frac{1}{2}
$$

in which case the operator $\mathscr{A}_{B}$ is denoted by $\mathscr{A}_{\rho \alpha}$ :

$$
\mathscr{A}_{B=2 \rho A^{\alpha}}=\mathscr{A}_{\rho \alpha}=\left|\begin{array}{cc}
0 & I \\
-A & -2 \rho A^{\alpha}
\end{array}\right| .
$$

At least in the special case $(2.1)$, the desired conclusion that $\exp \left(\mathscr{A}_{\rho \alpha} t\right)$ is differentiable on $E$ for all $t>0$ (i.e., that $\exp \left(\mathscr{A}_{\rho \alpha} t\right) E \subset \mathscr{D}\left(\mathscr{A}_{p \alpha}\right)$ ) is most directly obtained as a consequence of some special spectral properties enjoyed by the operator $\mathscr{A}_{\rho \alpha}$ which were noted in [C-T.1, C-T.2, T.2] (and, in fact, also for $\alpha>\frac{1}{2}$ ). In short, in the general situation where (2.5) below holds, the operator $\mathscr{A}_{\rho \alpha}$ is the direct (nonorthogonal) sum of two (explicitly identified) normal operators on $E$. For sake of brevity, we shall recall below only those facts which are strictly needed for our analysis of the present section and refer to [C-T.1, CT.2] for further information and justification. Without loss of generality, we can take that $A$ has compact resolvent for simplicity of exposition. Let $\left\{\mu_{n}\right\}_{n=1}^{\infty}$, $0<\mu_{1} \leq \mu_{2} \leq \mu_{3} \leq \cdots \rightarrow+\infty$ be the eigenvalues of the positive self-adjoint operator $A$, and let $\left\{e_{n}\right\}_{n=1}^{\infty}$ be its corresponding eigenvectors, $A e_{n}=\mu_{n} e_{n}$, subject to the normalization (2.4) below and forming a complete, orthogonal family on $X$. Then the eigenvalues $\left\{\lambda_{n}^{+,-}\right\}_{n=1}^{\infty}$ of the operator $\mathscr{A}_{\rho \alpha}$ in (2.2) (which has compact resolvent on $E$ ) are given by

$$
\lambda_{n}^{+,-}=\left(-\rho \pm \sqrt{\rho^{2}-\mu_{n}^{1-2 \alpha}}\right) \mu_{n}^{\alpha},
$$

with corresponding eigenvectors $\left\{\boldsymbol{\Phi}_{n}^{+,-}\right\}_{n=1}^{\infty}$ on $E$ (explicitly identified, e.g., [C-T.1, C-T.2]) normalized in $E$ :

$$
\left\|\Phi_{n}^{+,-}\right\|_{E}=1 \Leftrightarrow\left(\mu_{n}+\left|\lambda_{n}^{+}\right|^{2}\right)\left\|e_{n}\right\|_{X}^{2}=1 .
$$

Lemma 2.1. Let (2.1) hold. Then the strongly continuous semigroup of contractions $\exp \left(\mathscr{A}_{\rho \alpha} t\right)$ generated on $E$ by $\mathscr{A}_{\rho \alpha}$ in (2.2) (once closed) is differentiable on $E$ for all $t>0: \exp \left(\mathscr{A}_{\rho \alpha} t\right) E \subset \mathscr{D}\left(\mathscr{A}_{\rho \alpha}\right), t>0$, so that $\mathscr{A}_{\rho \alpha} \exp \left(\mathscr{A}_{\rho \alpha} t\right)$ is a bounded operator on all of $E$.

Proof. We shall rely heavily on spectral properties established in [C-T.1, C-T.2]. Here, under the assumption

$$
\rho^{2} \neq \mu_{n}^{1-2 \alpha} \text { for all } n, \text { so that } \lambda_{n}^{+} \neq \lambda_{n}^{-} \text {for all } n, \quad 0<\rho<\infty, 0<\alpha<\frac{1}{2},
$$


it is proved, e.g., [C-T.2, Lemma A.2], that the following expansion holds true for all $x \in E$ :

$$
x=\sum_{n=1}^{\infty}\left(x, \Phi_{n}^{*-}\right)_{E} \Phi_{n}^{+}+\sum_{n=1}^{\infty}\left(x, \Phi_{n}^{*+}\right)_{E} \Phi_{n}^{-},
$$

where $\left\{\Phi_{n}^{*+,-}\right\}$ are the (nonnormalized) eigenvectors of $\mathscr{A}_{\rho \alpha}^{*}$ (the adjoint of $\mathscr{A}_{\rho \alpha}$ in $E$ ), explicitly identified in [C-T.1, C-T.2], corresponding to the eigenvalues $\overline{\lambda_{n}^{+}}$and $\overline{\lambda_{n}^{-}}$of $\mathscr{A}_{\rho \alpha}^{*}$, which form a bi-orthogonal system with respect to the eigenvectors $\left\{\Phi_{n}^{+,-}\right\}$of $\mathscr{A}_{\rho \alpha}$, corresponding to its eigenvalues $\lambda_{n}^{+,-}$. Moreover, $\left\{\Phi_{n}^{+}\right\}_{n=1}^{\infty}$ and $\left\{\Phi_{n}^{-}\right\}_{n=1}^{\infty}$ are two orthogonal families on $E$ and $E=E^{+} \oplus E^{-}, E^{+} \cap E^{-}=\{0\} ; E^{+}=\overline{\operatorname{span}}\left\{\Phi_{n}^{+}\right\}_{n=1}^{\infty}$ and similarly for $E^{-}$. Thus, $\mathscr{A}_{\rho \alpha}$, restricted on $E^{+}$or on $E^{-}$, is a normal operator (but $\mathscr{A}_{\rho \alpha}$ is not a normal operator on $E$ ). With reference to (2.6) we have

$$
\begin{gathered}
\left(x, \Phi_{n}^{*-}\right)_{E}=\left(x^{+}, \Phi_{n}^{+}\right)_{E}, \quad\left(x, \Phi_{n}^{*+}\right)_{E}=\left(x^{-}, \Phi_{n}^{-}\right)_{E}, \\
x=x^{+}+x^{-}, \quad x^{+} \in E^{+}, x^{-} \in E^{-} .
\end{gathered}
$$

It then follows from (2.6) that

$$
\mathscr{A}_{\rho \alpha} e^{\mathscr{A}_{\rho} t} x=\sum_{n=1}^{\infty} \lambda_{n}^{+} e^{\lambda_{n}^{+} t}\left(x, \Phi_{n}^{*-}\right)_{E} \Phi_{n}^{+}+\sum_{n=1}^{\infty} \lambda_{n}^{-} e^{\lambda_{n}^{-} t}\left(x, \Phi_{n}^{*+}\right)_{E} \Phi_{n}^{-}
$$

and hence

$$
\begin{aligned}
& \left\|\mathscr{A}_{\rho \alpha} e^{\mathscr{A}_{\rho \alpha} t} x\right\|_{E}^{2} \\
& \quad \leq 2\left\{\sum_{n=1}^{\infty}\left|\lambda_{n}^{+}\right|^{2} e^{2\left(\operatorname{Re} \lambda_{n}^{+}\right) t}\left|\left(x, \Phi_{n}^{*-}\right)_{E}\right|^{2}+\sum_{n=1}^{\infty}\left|\lambda_{n}^{-}\right|^{2} e^{2\left(\operatorname{Re} \lambda_{n}^{-}\right) t}\left|\left(x, \Phi_{n}^{*+}\right)_{E}\right|^{2}\right\}
\end{aligned}
$$

Our goal is to show that $\mathscr{A}_{\rho \alpha} e^{\mathscr{A}_{\rho \alpha} t} \in \mathscr{L}(E), t>0$. To this end, we note that, for $0<\alpha<\frac{1}{2}$, we have $\mu_{n}^{1-2 \alpha}-\rho^{2}>0$ for all $n$ sufficiently large, hence, by (2.3),

$$
\lambda_{n}^{+,-}=\left(-\rho \pm i \sqrt{\mu_{n}^{1-2 \alpha}-\rho^{2}}\right) \mu_{n}^{\alpha}
$$

and thus

$$
\left|\lambda_{n}^{+,-}\right|^{2}=\mu_{n}, \quad \operatorname{Re} \lambda_{n}^{+,-}=-\rho \mu_{n}^{\alpha}, \quad 0<\alpha<\frac{1}{2}, \text { all } n \text { sufficiently large. }
$$

From here it follows readily that, for $t>0$,

$$
\left|\lambda_{n}^{+,-}\right|^{2} e^{2\left(\operatorname{Re} \lambda_{n}^{+-}-\right) t}=\mu_{n} e^{-2 \rho \mu_{n}^{\alpha} t} \leq C_{t, \alpha} \quad \text { uniformly in } n,
$$

where $C_{t, \alpha}$ is a positive function of $t$ and $\alpha$, but not of $n$ (since the function $f(x)=x \exp \left(-x^{\alpha} t\right)$, for $x \geq 0$ and $t>0$ fixed, has its maximum at $x^{\alpha}=$ $(1 / \alpha t))$. Then (2.10), (2.12) yield, by virtue also of (2.7), that

$$
\left\|\mathscr{A}_{\rho \alpha} e^{\mathscr{A}_{\rho a} t} x\right\|_{E}^{2} \leq C_{t, \alpha}\left(\left\|x^{+}\right\|_{E}^{2}+\left\|x^{-}\right\|_{E}^{2}\right)<\infty, \quad x \in E,
$$


and Lemma 2.1 follows from (2.13), at least under (2.5). However, any eigenvalue which violates $(2.5)$ has only a finite dimensional eigenspace which can be factored off. The proof is complete.

The known [C-T.1, C-T.2] expression of the resolvent $R\left(\lambda, \mathscr{A}_{B}\right)$ of $\mathscr{A}_{B}$ is

$$
\begin{gathered}
R\left(\lambda, \mathscr{A}_{B}\right)=\left[\begin{array}{cc}
\frac{I-V_{B}^{-1}(\lambda) A}{\lambda} & V_{B}^{-1}(\lambda) \\
-V_{B}^{-1}(\lambda) A & \lambda V_{B}^{-1}(\lambda)
\end{array}\right] \\
V_{B}(\lambda)=\lambda^{2} I+\lambda B+A=V_{\rho \alpha}(\lambda)+\lambda\left(B-2 \rho A^{\alpha}\right) \\
V_{\rho \alpha}(\lambda)=\lambda^{2} I+\lambda 2 \rho A^{\alpha}+A,
\end{gathered}
$$

valid at least for $\operatorname{Re} \lambda>0$. Lemma 2.1 and the known characterization [P.1, Theorem 4.8, p. 57] of differentiable semigroups imply the following necessary condition for the resolvent $R\left(\lambda, \mathscr{A}_{\rho \alpha}\right)$ when $B=2 \rho A^{\alpha}, 0<\alpha<\frac{1}{2}$.

Corollary 2.2. Let the assumptions of Lemma 2.1 hold true with $\rho$ and $\alpha$ fixed, $0<\alpha<\frac{1}{2}$. Then, for every $b>0$, there are constants $a_{b}$ real and $C_{b}$ positive (depending also on $\rho$ and $\alpha$, but we shall omit such indication) such that

$$
\begin{gathered}
\text { resolvent set of } \mathscr{A}_{\rho \alpha} \supset \Sigma_{b}=\left\{\lambda: \operatorname{Re} \lambda>a_{b}-b \log |\operatorname{Im} \lambda|\right\} \\
\left\|R\left(\lambda, \mathscr{A}_{\rho \alpha}\right)\right\|_{\mathscr{L}(E)} \leq C_{b}|\operatorname{Im} \lambda|, \quad \lambda \in \Sigma_{b}, \operatorname{Re} \lambda \leq 0 .
\end{gathered}
$$

For the purposes of our main Theorem 1.1, property (2.18)-as well as its readily obtainable equivalent version in terms of the entries of the matrix $R\left(\lambda, \mathscr{A}_{\rho \alpha}\right)$ in $(2.14)$-appears to be inadequate. We need to take advantage of the property of the eigenvalues $\lambda_{n}^{+,-}$in (2.11) that the function $\left|\operatorname{Re} \lambda_{n}^{+,-}\right| \rightarrow\left|\operatorname{Im} \lambda_{n}^{+,-}\right|$displays only a polynomial growth (for fixed $\alpha<\frac{1}{2}$ ), a much better behavior than the exponential growth allowed for the general class of differentiable semigroups. More precisely, from (2.11) we have (with notation $a_{n} \sim b_{n}$ meaning $b_{n} k \leq a_{n} \leq K b_{n}$ as $n \rightarrow \infty, 0<k<K<\infty$ independent of $n$, as usual)

$$
\left|\operatorname{Re} \lambda_{n}^{+,-}\right|=\rho \mu_{n}^{\alpha}, \quad\left|\operatorname{Im} \lambda_{n}^{+,-}\right| \sim \mu_{n}^{1 / 2} \sim\left(\mu_{n}^{\alpha}\right)^{1 / 2 \alpha} \sim\left|\operatorname{Re} \lambda_{n}^{+,-}\right|^{1 / 2 \alpha} \text { as } n \rightarrow \infty \text {. }
$$

This fact will imply the following stronger property for $R\left(\lambda, \mathscr{A}_{\rho \alpha}\right)$ which will be crucial for our subsequent analysis. To prove Gevrey class, differentiability for $\exp \left(\mathscr{A}_{B} t\right)$ on $E$, we shall in fact make use of the sufficient condition as in [T.4, Theorem 4; P.1, Theorem 4.9, p. 57] evaluated on the imaginary axis $\lambda=i \tau, \tau \in \mathbf{R}$ (since $\exp \left(\mathscr{A}_{B} t\right)$ is a contraction semigroup on $E$ ). Thus, we shall express the following result only for $\lambda=i \tau, \tau \in \mathbf{R}$, the case which is needed below. 
Corollary 2.3. Let $0<\alpha<\frac{1}{2}$ be fixed, as in Lemma 2.1 .

(i) Then there exists a constant $C_{\rho \alpha}>0$ such that the following bound holds true uniformly in $\tau \in \mathbf{R}$ :

$$
\left\||\tau|^{2 \alpha} R\left(i \tau, \mathscr{A}_{\rho \alpha}\right)\right\|_{\mathscr{L}(E)} \leq C_{\rho \alpha} .
$$

(ii) Equivalently, since $E=\mathscr{D}\left(A^{1 / 2}\right) \times X$, there is a constant $C_{\rho \alpha}>0$ such that the following estimates hold true uniformly in $|\tau| \geq 1$ :

$$
\left.\begin{array}{l}
\left\||\tau|^{2 \alpha-1} A V_{\rho \alpha}^{-1}(i \tau)\right\|_{\mathscr{L}(X)} \\
\left\||\tau|^{2 \alpha} A^{1 / 2} V_{\rho \alpha}^{-1}(i \tau)\right\|_{\mathscr{L}(X)} \\
\left\||\tau|^{2 \alpha+1} V_{\rho \alpha}^{-1}(i \tau)\right\|_{\mathscr{L}(X)}
\end{array}\right\} \leq C_{\rho \alpha} .
$$

(iii) We interpolate between (2.21) and (2.22) and between (2.22) and (2.23) to obtain, respectively, the following results: for any $0 \leq \theta \leq 1$, there is a constant $C_{\rho \alpha \theta}>0$ such that the following bounds hold true, uniformly in $|\tau| \geq 1$ :

$$
\begin{gathered}
\left\||\tau|^{2 \alpha-\theta} A^{\theta / 2} A^{1 / 2} V_{\rho \alpha}^{-1}(i \tau)\right\|_{\mathscr{L}(X)} \leq C_{\rho \alpha \theta} \\
\left\||\tau|^{2 \alpha+1-\theta} A^{\theta / 2} V_{\rho \alpha}^{-1}(i \tau)\right\|_{\mathscr{L}(X)} \leq C_{\rho \alpha \theta} .
\end{gathered}
$$

Remark 2.1. For future easy reference, we single out the explicit versions of (2.24) and (2.25) for $\theta=\alpha$. These will be the only cases which will be invoked in our subsequent analysis. There is a constant $C_{\rho \alpha}>0$ such that the following bounds hold true uniformly in $|\tau| \geq 1$ :

$$
\begin{gathered}
\left\||\tau|^{\alpha} A^{(1+\alpha) / 2} V_{\rho \alpha}^{-1}(i \tau)\right\|_{\mathscr{L}(X)} \leq C_{\rho \alpha} \\
\left\||\tau|^{1+\alpha} A^{\alpha / 2} V_{\rho \alpha}^{-1}(i \tau)\right\|_{\mathscr{L}(X)} \leq C_{\rho \alpha} .
\end{gathered}
$$

Proof of Corollary 2.3. (i). There is a $\varepsilon_{\alpha}>0$ such that

$$
\left|i \tau-\lambda_{n}^{+,-}\right| \geq \varepsilon_{\alpha}|\tau|^{2 \alpha}, \quad \text { uniformly in } \tau \in \mathbf{R} \text { with } n=1,2, \ldots
$$

To show (2.28), let, say, $\tau>0$ be given, in which case it suffices to consider only $\left\{\lambda_{n}^{+}\right\}_{n=1}^{\infty}$. In the quadrant $(r, \tau)$ below, the eigenvalues $\lambda_{n}^{+}$may be taken to lie on the curve $\tau=(r)^{1 / 2 \alpha}$ or $r=\tau^{2 \alpha}$ (from (2.19)). We thus have

$$
\left|i \tau-\lambda_{n}^{+}\right|^{2}=\left|\tau-\operatorname{Im} \lambda_{n}^{+}\right|^{2}+\left[\left(\operatorname{Im} \lambda_{n}^{+}\right)^{2 \alpha}\right]^{2} .
$$

Let $0<\varepsilon<1$ be given. If $\operatorname{Im} \lambda_{n}^{+} \geq \varepsilon \tau$, then

$$
\left|i \tau-\lambda_{n}^{+}\right| \geq\left(\operatorname{Im} \lambda_{n}^{+}\right)^{2 \alpha} \geq \varepsilon^{2 \alpha} \tau^{2 \alpha}
$$




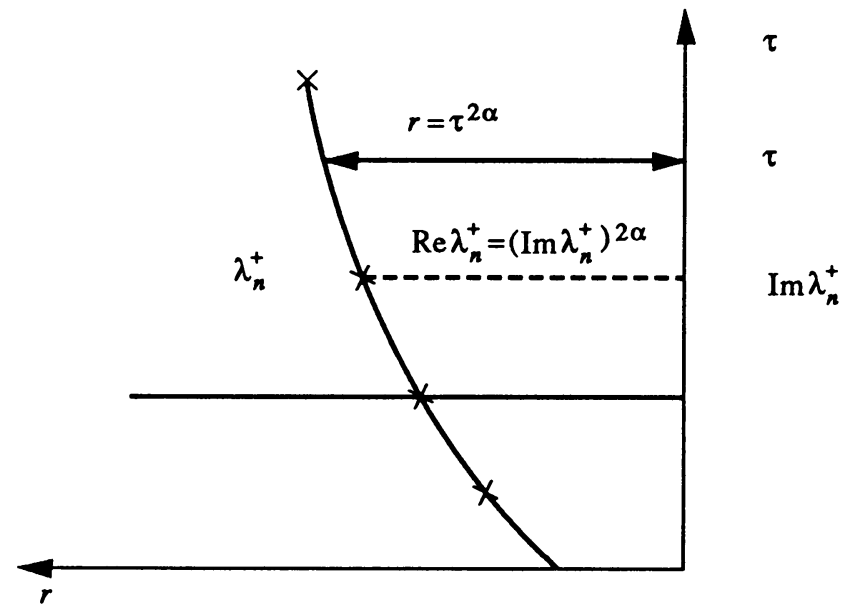

as desired. If, instead, $\left(\operatorname{Im} \lambda_{n}^{+}\right)<\varepsilon \tau$, then for $\tau \geq 1$,

$$
\left|i \tau-\lambda_{n}^{+}\right| \geq\left|\tau-\operatorname{Im} \lambda_{n}^{+}\right| \geq(1-\varepsilon) \tau \geq(1-\varepsilon) \tau^{2 \alpha}
$$

since $0<2 \alpha<1$ as desired. Next, from (2.6), (2.7) we obtain, at least for $\operatorname{Re} \lambda \geq 0$,

$$
R\left(\lambda, \mathscr{A}_{\rho \alpha}\right) x=\sum_{n=1}^{\infty} \frac{\left(x^{+}, \Phi_{n}^{+}\right)_{E}}{\lambda-\lambda_{n}^{+}} \Phi_{n}^{+}+\sum_{n=1}^{\infty} \frac{\left(x^{-}, \Phi_{n}^{-}\right)_{E}}{\lambda-\lambda_{n}^{-}} \Phi_{n}^{-}
$$

so that, for $\lambda=i \tau$, we have

$$
\begin{aligned}
\left\|R\left(i \tau, \mathscr{A}_{\rho \alpha}\right) x\right\|_{E}^{2} & \leq 2\left\{\sum_{n=1}^{\infty} \frac{\left|\left(x^{+}, \Phi_{n}^{+}\right)_{E}\right|^{2}}{\left|i \tau-\lambda_{n}^{+}\right|^{2}}+\sum_{n=1}^{\infty} \frac{\left|\left(x^{-}, \Phi_{n}^{-}\right)_{E}\right|^{2}}{\left|i \tau-\lambda_{n}^{-}\right|^{2}}\right\} \\
(\text { from }(2.28)) & \leq \frac{1}{\varepsilon_{\alpha}^{2}|\tau|^{4 \alpha}}\left\{\sum_{n=1}^{\infty}\left|\left(x^{+}, \Phi_{n}^{+}\right)_{E}\right|^{2}+\sum_{n=1}^{\infty}\left|\left(x^{-}, \Phi_{n}^{-}\right)_{E}\right|^{2}\right\} \\
& =\frac{1}{\varepsilon_{\alpha}^{2}|\tau|^{4 \alpha}}\left(\left\|x^{+}\right\|_{E}^{2}+\left\|x^{-}\right\|_{E}^{2}\right)<\infty,
\end{aligned}
$$

and (2.31) yields the desired conclusion (2.20) for $\tau^{2 \alpha} R\left(i \tau, \mathscr{A}_{\rho \alpha}\right)$.

(ii). Since, as in [C-T.2],

$\left\|R\left(\lambda, \mathscr{A}_{B}\right)\right\|_{\mathscr{L}(E)}=\left\|\left[\begin{array}{cc}A^{1 / 2} & 0 \\ 0 & I\end{array}\right]\left[\begin{array}{cc}\frac{I-V_{B}^{-1}(\lambda) A}{\lambda} & V_{B}^{-1}(\lambda) \\ -V_{B}^{-1}(\lambda) A & \lambda V_{B}^{-1}(\lambda)\end{array}\right]\left[\begin{array}{cc}A^{-1 / 2} & 0 \\ 0 & I\end{array}\right]\right\|_{\mathscr{L}(W)}$,

$W=X \times X$, then (2.20) plainly yields inequalities (2.21)-(2.23) via (2.32) used for $B=2 \rho A^{\alpha}$. 
(iii). We use the interpolation (moment) inequality [L-M.1, p. 19; K.1, p. 115]. To interpolate between (2.21) and (2.22) we set

$$
\Lambda=\frac{A^{1 / 2}}{|\tau|} \quad \text { and } \quad u=A^{1 / 2} V_{\rho \alpha}^{-1}(i \tau) x
$$

so that $\left\|\Lambda^{\theta} u\right\| \leq\|\Lambda u\|^{\theta}\|u\|^{1-\theta}$ in the norm of $\mathscr{L}(X)$ gives

$$
\left\|\left(|\tau|^{-1} A^{1 / 2}\right)^{\theta} A^{1 / 2} V_{\rho \alpha}^{-1}(i \tau)\right\|_{\mathscr{L}(X)} \leq\left\||\tau|^{-1} A V_{\rho \alpha}^{-1}(i \tau)\right\|_{\mathscr{L}(X)}^{\theta}\left\|A^{1 / 2} V_{\rho \alpha}^{-1}(i \tau)\right\|_{\mathscr{L}(X)}^{1-\theta} .
$$

Multiplying (2.34) through by $|\tau|^{2 \alpha}=\left(|\tau|^{2 \alpha}\right)^{\theta}\left(|\tau|^{2 \alpha}\right)^{1-\theta}$ yields (2.24) via (2.21) and (2.22), as desired. The proof of (2.25) by interpolating between (2.22) and (2.23) is similar: now we set $\Lambda$ as in (2.33) and $u=|\tau| V_{\rho \alpha}^{-1}(i \tau) x$.

Remark 2.2. For general $A$ which does not have compact resolvent, one employs the self-adjoint calculus with integrals replacing the sums above.

\section{Proof of Theorem 1.1}

Our main goal is to establish that the strongly continuous contraction semigroup $\exp \left(\mathscr{A}_{B} t\right)$ satisfies on $E$ the relation

$$
\limsup _{|\tau| \rightarrow \infty}|\tau|^{2 \alpha}\left\|R\left(i \tau, \mathscr{A}_{B}\right)\right\|_{\mathscr{L}(E)}=C<\infty, \quad \tau \in \mathbf{R}
$$

Once (3.1) is established, we invoke the sufficient condition as in [T.4, Theorem 4] and conclude that $\exp \left(\mathscr{A}_{B} t\right)$ is of Gevrey class $\delta>1 / 2 \alpha$ on $E$ for all $t>0$.

Using (2.32), we readily see that condition (3.1) is equivalent to the following set of three equalities in the norm of $\mathscr{L}(X)$ :

$$
\begin{gathered}
\varlimsup_{|\tau| \rightarrow \infty}|\tau|^{2 \alpha-1}\left\|A^{1 / 2} V_{B}^{-1}(i \tau) A^{1 / 2}\right\|=C<\infty, \\
\varlimsup_{|\tau| \rightarrow+\infty}|\tau|^{2 \alpha}\left\|A^{1 / 2} V_{B}^{-1}(i \tau)\right\|=C<\infty, \\
\varlimsup_{|\tau| \rightarrow+\infty}|\tau|^{2 \alpha+1}\left\|V_{B}^{-1}(i \tau)\right\|=C<\infty .
\end{gathered}
$$

In fact, let $a_{i j}, i, j=1,2$, denote the entries of the operator matrix $R\left(i \tau, \mathscr{A}_{B}\right)$ as in (2.14). Then (3.2), (3.3), and (3.4) refer directly to $a_{11}, a_{12}$, and $a_{22}$, while $a_{21}$ yields

$$
\varlimsup_{|\tau| \rightarrow+\infty}|\tau|^{2 \alpha}\left\|V_{B}^{-1}(i \tau) A^{1 / 2}\right\|=C<\infty,
$$

which is equivalent to (3.3) since

$$
\left(V_{B}^{-1}(i \tau) A^{1 / 2}\right)^{*}=A^{1 / 2} V_{B}^{-1}(\overline{i \tau})=A^{1 / 2} V_{B}^{-1}(-i \tau) .
$$

Thus, our task is to establish (3.2), (3.3), (3.4). This we shall do next. 


\subsection{Proof of (3.2). Since}

$$
V_{\rho \alpha}^{-1}(i \tau)-V_{B}^{-1}(i \tau)=V_{\rho \alpha}^{-1}(i \tau)\left(V_{B}(i \tau)-V_{\rho \alpha}(i \tau)\right) V_{B}^{-1}(i \tau),
$$

we compute via $V_{B}(i \tau)-V_{\rho \alpha}(i \tau)=i \tau\left(B-2 \rho A^{\alpha}\right)($ see $(2.15))$ :

$$
\begin{aligned}
& |\tau|^{2 \alpha-1}\left(A^{1 / 2} V_{\rho \alpha}^{-1}(i \tau) A^{1 / 2}-A^{1 / 2} V_{B}^{-1}(i \tau) A^{1 / 2}\right) \\
& \quad=i \tau|\tau|^{2 \alpha-1} A^{1 / 2+\alpha / 2} V_{\rho \alpha}^{-1}(i \tau)\left(S_{\alpha}-2 \rho I\right) A^{\alpha / 2} V_{B}^{-1}(i \tau) A^{1 / 2},
\end{aligned}
$$

where we have set

$$
S_{\alpha}=A^{-\alpha / 2} B A^{-\alpha / 2} \in \mathscr{L}(X)
$$

and $S_{\alpha}$ is a bounded operator on $X$, boundedly invertible here, by assumption (1.9a-b) (selfadjoint if $B$ is selfadjoint). We now invoke (2.21) and (2.26) for the terms $|\tau|^{2 \alpha-1} \cdot A^{1 / 2} V_{\rho \alpha}^{-1}(i \tau) A^{1 / 2}$ and $|\tau|^{\alpha} A^{1 / 2+\alpha / 2} V_{\rho \alpha}^{-1}(i \tau)$ in (3.7). Thus (3.7) reveals that the limit

$$
\varlimsup_{|\tau| \rightarrow \infty}|\tau|^{2 \alpha-1}\left\|A^{1 / 2} V_{B}^{-1}(i \tau) A^{1 / 2}\right\|=C<\infty,
$$

i.e. (3.2), holds true, as soon as we show the following bound, uniformly in $|\tau| \geq 1$ :

$$
|\tau|^{\alpha}\left\|A^{\alpha / 2} V_{B}^{-1}(i \tau) A^{1 / 2}\right\| \leq C_{\rho \alpha} .
$$

To show (3.10) we compute via (3.6), (2.15), and (3.8):

$$
\begin{aligned}
& A^{\alpha / 2} V_{\rho \alpha}^{-1}(i \tau) A^{1 / 2}-A^{\alpha / 2} V_{B}^{-1}(i \tau) A^{1 / 2}=i \tau A^{\alpha} V_{\rho \alpha}^{-1}(i \tau)\left(S_{\alpha}-2 \rho I\right) A^{\alpha / 2} V_{B}^{-1}(i \tau) A^{1 / 2} \\
& \text { or }
\end{aligned}
$$

$$
|\tau|^{\alpha} A^{\alpha / 2} V_{\rho \alpha}^{-1}(i \tau) A^{1 / 2}=\left[I+i \tau A^{\alpha} V_{\rho \alpha}^{-1}(i \tau)\left(S_{\alpha}-2 \rho I\right)\right]|\tau|^{\alpha} A^{\alpha / 2} V_{B}^{-1}(i \tau) A^{1 / 2}
$$

But a fortiori from (2.26) we have that the left-hand side of (3.12) is uniformly bounded in $|\tau| \geq 1$. Thus, we see from (3.12) that to establish (3.10), it suffices to invoke

Lemma 3.1. Let $0<2 \rho<\rho_{1}$, see (1.5). Then the operator

$$
\mathscr{G}_{\rho \alpha}(i \tau)=I+i \tau A^{\alpha} V_{\rho \alpha}^{-1}(i \tau)\left(S_{\alpha}-2 \rho I\right)
$$

is boundedly invertible on $\mathscr{L}(X)$ (isomorphism), uniformly in $\tau \in \mathbf{R}$; in particular there is a positive constant $C_{\rho \alpha}$ such that, for all $\tau \in \mathbf{R}$, we have

$$
\left\|\mathscr{G}_{\rho \alpha}^{-1}(i \tau)\right\|_{\mathscr{L}(X)}=\left\|\left[I+i \tau A^{\alpha} V_{\rho \alpha}^{-1}(i \tau)\left(S_{\alpha}-2 \rho I\right)\right]^{-1}\right\|_{\mathscr{L}(X)} \leq C_{\rho \alpha} .
$$

Proof. See Lemma 4.2 of [C-T.2] for $B$ positive selfadjoint operator satisfying (1.5). The extension of the proof in this reference to $B$ which satisfies $\left(\right.$ H. $\left.3^{\prime}\right)$ in (1.9a-b) requires only a minor variation in going from (4.19) to (4.20) in [CT.2]. Now the operator $Q=S_{\alpha}-2 \rho I$ is bounded by (1.9), injective, and can 
be written as $Q=Q_{1}+i Q_{2}$, with $Q_{1}$ positive selfadjoint and $Q_{2}$ selfadjoint. Then, if we now take real part and imaginary part of $(Q x, x)$, we see that (4.20) in [C-T.2] becomes

$$
\operatorname{Re}\left(Q^{-1} x, x\right) \geq\left(Q_{1} Q^{-1} x, Q^{-1} x\right) \geq \frac{\rho_{1}-2 \rho}{\left\|Q^{-1}\right\|}\|x\|^{2}
$$

and Lemma 4.2 in [C-T.2] is proved for $B$ satisfying (1.9a-b).

Thus (3.10) follows, and hence (3.9) is established. Equation (3.2) is proved.

3.2. Proof of (3.3). We compute, from (3.6), (2.15), and (3.8),

$$
\begin{aligned}
& |\tau|^{2 \alpha}\left(A^{1 / 2} V_{\rho \alpha}^{-1}(i \tau)-A^{1 / 2} V_{B}^{-1}(i \tau)\right) \\
& \quad=i|\tau|^{\alpha} A^{1 / 2+\alpha / 2} V_{\rho \alpha}^{-1}(i \tau)\left(S_{\alpha}-2 \rho I\right) \tau|\tau|^{\alpha} A^{\alpha / 2} V_{B}^{-1}(i \tau) .
\end{aligned}
$$

We now invoke (2.22) and again (2.26) for the terms $|\tau|^{2 \alpha} A^{1 / 2} V_{\rho \alpha}^{-1}(i \tau)$ and $|\tau|^{\alpha} A^{1 / 2+\alpha / 2} V_{\rho \alpha}^{-1}(i \tau)$ in (3.15). Thus (3.15) reveals that the limit

$$
\varlimsup_{|\tau| \rightarrow \infty}|\tau|^{2 \alpha}\left\|A^{1 / 2} V_{B}^{-1}(i \tau)\right\|=C<\infty,
$$

i.e (3.3), holds true, as soon as we show the following bound, uniformly in $|\tau| \geq 1$ :

$$
|\tau|^{\alpha+1}\left\|A^{\alpha / 2} V_{B}^{-1}(i \tau)\right\| \leq C_{\rho \alpha} .
$$

To show (3.17) compute via (3.6), (2.15), and (3.8):

$$
\begin{aligned}
& |\tau|^{\alpha+1} A^{\alpha / 2} V_{\rho \alpha}^{-1}(i \tau)-|\tau|^{\alpha+1} A^{\alpha / 2} V_{B}^{-1}(i \tau) \\
& \quad=i \tau A^{\alpha} V_{\rho \alpha}^{-1}(i \tau)\left(S_{\alpha}-2 \rho I\right)|\tau|^{\alpha+1} A^{\alpha / 2} V_{B}^{-1}(i \tau)
\end{aligned}
$$

or

$$
|\tau|^{\alpha+1} A^{\alpha / 2} V_{\rho \alpha}^{-1}(i \tau)=\left[I+i \tau A^{\alpha} V_{\rho \alpha}^{-1}(i \tau)\left(S_{\alpha}-2 \rho I\right)\right]|\tau|^{\alpha+1} A^{\alpha / 2} V_{B}^{-1}(i \tau),
$$

where the left-hand side of (3.19) is uniformly bounded in $|\tau| \geq 1$, a fortiori from (2.27). Thus recalling Lemma 3.1 on (3.19) yields (3.17). Hence, (3.16) is established and so is (3.3).

3.3. Proof of (3.4). From (3.6), (2.15), (3.8) we compute

$$
\begin{aligned}
& |\tau|^{2 \alpha+1}\left(V_{\rho \alpha}^{-1}(i \tau)-V_{B}^{-1}(i \tau)\right) \\
& \quad=|\tau|^{\alpha+1} A^{\alpha / 2} V_{\rho \alpha}^{-1}(i \tau)\left(S_{\alpha}-2 \rho I\right) i \tau|\tau|^{\alpha} A^{\alpha / 2} V_{B}^{-1}(i \tau) .
\end{aligned}
$$

We now invoke the limits (2.23) and (2.27) for the terms $|\tau|^{2 \alpha+1} V_{\rho \alpha}^{-1}(i \tau)$ and 
$|\tau|^{\alpha+1} A^{\alpha / 2} V_{\rho \alpha}^{-1}(i \tau)$ in (3.20), and the uniform bound (3.17) for the term $|\tau|^{\alpha+1} \times$ $A^{\alpha / 2} V_{B}^{-1}(i \tau)$ in (3.20). Thus (3.20) reveals that the limit

$$
\varlimsup_{|\tau| \rightarrow \infty}|\tau|^{2 \alpha+1}\left\|V_{B}^{-1}(i \tau)\right\|=C<\infty,
$$

i.e. (3.4), holds true. The proof of Theorem 1.1 is complete.

\section{EXAMPLES}

A simple example where the above Theorem 1.1 applies with $\alpha=\frac{1}{3}$ is the following problem in the unknown $w(t, x)$ defined on a smooth bounded domain $\Omega$ of $\mathbf{R}^{n}$ :

$$
\begin{cases}w_{t t}-\Delta^{3} w-\rho \Delta w_{t}=0 & \text { in }(0, T] \times \Omega \equiv Q \\ w(0, x)=w_{0}, w_{t}(0, x)=w_{1} & \text { in } \Omega \\ \left.w\right|_{\Sigma}=\left.\Delta w\right|_{\Sigma}=\left.\Delta^{2} w\right|_{\Sigma} \equiv 0 & \text { in }(0, T] \times \Gamma \equiv \Sigma\end{cases}
$$

where $\rho>0$ is a constant. Here, $A f=-\Delta^{3} f, \mathscr{D}(A)=\left\{f \in H^{6}(\Omega):\left.f\right|_{\Gamma}=\right.$ $\left.\left.\Delta f\right|_{\Gamma}=\left.\Delta^{2} f\right|_{\Gamma}=0\right\}$ and $A^{1 / 3} f=-\Delta f, \mathscr{D}\left(A^{1 / 3}\right)=\left\{f \in H^{2}(\Omega):\left.f\right|_{\Gamma}=0\right\}$. Hence, the abstract version of problem (4.1) is given by the equation $\ddot{w}+A \dot{w}+$ $\rho A^{1 / 3} \dot{w}=0$, to which Theorem 1.1 applies with $\alpha=\frac{1}{3}$. Other examples can readily be constructed in a similar way. Problems with higher order operators $(-\Delta)^{q}$ are considered in J. L. Lions [L.2, §3.8].

\section{REFERENCES}

[B.1] A. V. Balakrishnan, Damping operators in continuum models of flexible structures, preprint.

[C-R.1] G. Chen and D. L. Russell, A mathematical model for linear elastic systems with structural damping, Quart. Appl. Math. 39 (1982), 433-454.

[C-T.1] S. Chen and R. Triggiani, Proof of two conjectures of G. Chen and D. L. Russell on structural damping for elastic systems: the case $\alpha=\frac{1}{2}$, in Springer-Verlag Lecture Notes in Math., vol. 1354, pp. 234-256 (Proceedings of the Seminar in Approximation and Optimization, University of Habana, Cuba, January 10-12, 1987).

[C-T.2] _ Proof of extensions of two conjectures on structural damping for elastic systems: the case $\frac{1}{2} \leq \alpha \leq 1$, Pacific J. Math. 39 (1989), 15-55.

[C-T.3] S. Chen and R. Triggiani, Analyticity of elastic systems with non selfadjoint dissipation: the general case, preprint, 1989.

[K.1] S. G. Krein, Linear differential equations in Banach space, Trans. Amer. Math. Soc. 29 (1971).

[K.2] T. Kato, Perturbation theory for linear operators, Springer-Verlag, 1966.

[L.1] W. Littman, A generalization of a theorem of Datko and Pazy, University of Minnesota, preprint, 1988; presented at International Conference, Baton Rouge, Louisiana, October 19-21, 1988.

[L.2] J. L. Lions, Controlabilite exacte des systemes distribues, Masson, Paris, 1989.

[L-M.1] J. L. Lions and E. Magenes, Non homogeneous boundary value problems and applications I, Springer-Verlag, New York, 1972.

[P.1] A. Pazy, Semigroups of linear operators and applications to partial differential equations, Springer-Verlag, New York, 1983. 
[T.1] R. Triggiani, On the stabilizability problem in Banach space, J. Math. Anal. Appl. 52 (1975), 383-403. Addendum J. Math. Anal. Appl. 56 (1976), 492-493.

[T.2] _ Improving stability properties of hyperbolic damped equations by boundary feedback, Springer-Verlag Lecture Notes (LNCIS), 1985, 400-409.

[T.3] __ Finite rank, relatively bounded perturbations of semi-group generators, Part III: a sharp result on the lack of uniform stabilization, Differential and Integral Equations 3 (1990), 503522.

[T.4] S. Taylor, Ph.D. thesis, Chapter 'Gevrey semigroups', School of Mathematics, University of Minnesota, 1989.

Department of Mathematics, Zhejiang University, Hangzhou, China

Department of Applied Mathematics, University of Virginia, Charlottesville, VirGINIA 22903 